\title{
Computing Equilibria in Dynamic Models with Occasionally Binding Constraints*
}

\author{
Johannes Brumm ${ }^{1}$ and Michael Grill ${ }^{1}$ \\ ${ }^{1}$ University of Mannheim
}

July 2, 2010

\begin{abstract}
We propose a method to compute equilibria in dynamic models with several continuous state variables and occasionally binding constraints. These constraints induce non-differentiabilities in policy functions. We develop an interpolation technique that addresses this problem directly: It locates the non-differentiabilities, and adds interpolation nodes there. To handle this flexible grid, it uses simplicial interpolation. Hence, we call this method Adaptive Simplicial Interpolation (ASI). We embed ASI into a time iteration algorithm to compute recursive equilibria in an infinite horizon endowment economy where heterogeneous agents trade in a bond and a stock subject to various trading constraints. We show that this method computes equilibria accurately and outperforms equidistant grid schemes by far.

Keywords: Adaptive Grid, Delaunay Interpolation, DSGE Models, Non-Differentiabilities, Occasionally Binding Constraints, Simplicial Interpolation, Trading Constraints JEL Classification: C63, C68, E21, G11
\end{abstract}

*We have benefited greatly from comments by Klaus Adam, Wouter den Haan, Ken Judd, Philip Jung, Tom Krebs, Dirk Krueger, Lars Ljungqvist and Albert Marcet. Special thanks go to Felix Kubler and Karl Schmedders for many helpful discussions. All remaining errors are ours. Contact Details: johannes.brumm@googlemail.com, michael_grill@gmx.de 


\section{Introduction}

In many applications of dynamic stochastic (general) equilibrium models, it is a natural modeling choice to include constraints that are occasionally binding. Examples are models with borrowing constraints, liquidity constraints, a zero bound on the nominal interest rate, or irreversible investments. These constraints induce non-differentiabilities in the policy functions, which make it challenging to compute equilibria. In particular, standard interpolation techniques using non-adaptive grids perform poorly both in terms of accuracy and shape of the computed policy function (see Judd et al. (2003)). This paper proposes a method that overcomes these problems, even for models with several continuous state variables. We call this method Adaptive Simplicial Interpolation (ASI). Its working principle is to locate the non-differentiablilites that are induced by occasionally binding constraints, and to use them as additional interpolation nodes. In what follows, the set of points at which the policy function fails to be differentiable due to the impact of a certain constraint is called a kink.

To present our algorithm, we consider a dynamic endowment economy where three or four (types of) agents face aggregate and idiosyncratic risk. To explain the main features of ASI we first compute equilibria in a simple two period version where agents trade in a bond subject to an ad-hoc borrowing constraint. Second, we embed ASI into a time iteration algorithm to solve an infinite horizon version of the model. Finally, we add a stock to the economy, replace the ad-hoc borrowing constraint by a collateral constraint, which depends on endogenous variables, and introduce a short sale constraint on the stock. Hence, short positions in the bond need to be collateralized by stock holdings, while the stock may not be shorted.

Compared to earlier papers using a similar setup, such as Heaton and Lucas (1996), den Haan (2001) or Kubler and Schmedders (2003), the model we consider differs in two respects, which both make it harder to compute equilibria: First, we solve models with more agents, which results in a continuous state space of higher dimension. As the kinks naturally form hypersurfaces in the state space, they are of higher dimension as well. Second, in our extension, the trading constraints that agents face depend on an endogenously determined 

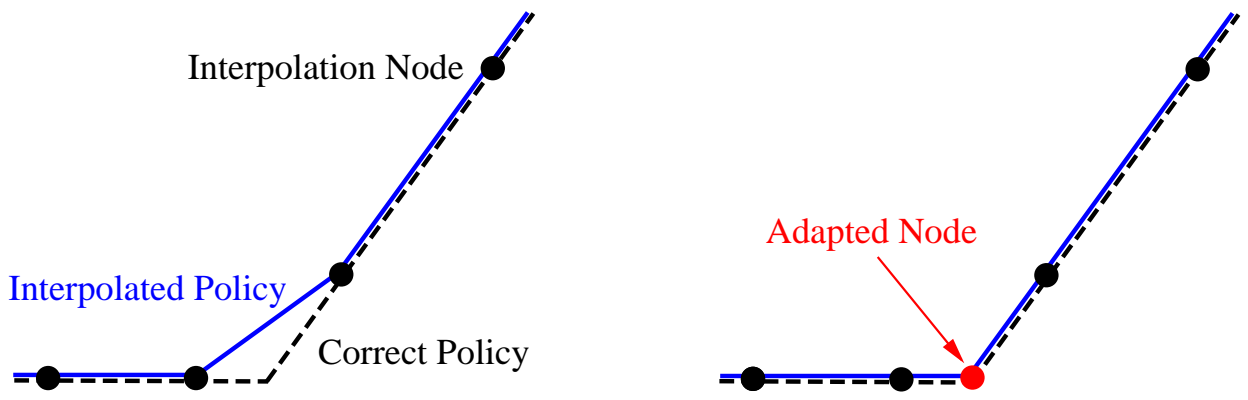

Figure 1: Non-adaptive (lhs) and adaptive (rhs) linear interpolation in 1D

variable, namely tomorrow's equilibrium price of the stock. Consequently, it is much harder to locate the kink, and ad hoc methods fail.

To illustrate the working principle of ASI, consider a simple one dimensional policy function with a kink, as displayed by the dashed line in Figure 1. Suppose this function is approximated by linear interpolation between equidistant grid points. The resulting interpolated policy is displayed as a solid line. Clearly, the approximation error is huge around the kink, and this is just because there is no interpolation node near the kink. If we knew the location of the kink and put a node there, then the approximation would be much better, as the right hand side of Figure 1 shows. This is the motivation for ASI, which directly addresses the problem of kinks in policy functions by placing additional grid points, called adapted points, at these non-differentiabilities. Unfortunatelly, in higher dimensional state spaces and with complex constraints, this is not as simple as Figure 1 suggests. Hence, we need a flexible interpolation technique and a systematic adaptation procedure.

To place grid points wherever needed, we use Delaunay interpolation, which consists of two steps. First, the convex hull of the set of grid points is covered with simplices, which results in a so called tessellation. Then we linearly interpolate locally on each simplex ${ }^{1}$.

We adapt the grid by placing grid points at the kinks, as follows: First, we solve the system of equilibrium conditions on an exogenous grid. Second, we use these

\footnotetext{
${ }^{1}$ Clearly, linear simplicial interpolation is only $C^{0}$ at the boundaries. For our purposes, this is desirable, because it provides a better fit at the kinks, and it makes the convergence behavior more stable.
} 
solutions to determine which edges of the tessellation cross kinks. Third, on each of these edges, we compute the point of intersection with the kink. Finally, we place a new grid point there.

Using this procedure with state spaces of more than one dimension, we get several adapted grid points for each kink. Delaunay tessellation connects these points by edges, such that the kinks are matched precisely.

To solve the above described infinite horizon model(s), we embed adaptive simplicial interpolation in a standard time iteration algorithm as explained in Judd (1992). To assess the accuracy of the computed equilibria, we follow Judd (1992) in calculating relative errors in Euler equations, subsequently called Euler errors. Concerning the measured Euler errors, we find that our method accurately computes equilibria for the two economies considered, and for reasonable and extreme calibrations of our model. Furthermore, we assess the relative performance of the adaptive grid scheme by comparing it to a standard equidistant grid scheme holding the interpolation technique fixed. We find that the adaptive grid scheme dominates by far: One needs to increase the number of equidistant grid points, and thereby CPU time, by more than two orders of magnitude in order to reach the high accuracy of the adaptive grid scheme.

In the literature, many algorithms have been applied to dynamic models with occasionally binding constraints. However, none of the existing algorithms addresses the problems of non-differentiabilities directly. Christiano and Fisher (2000) compared how several algorithms compute equilibria in a one sector growth model with irreversible investment. Hence, there is only one continuous state variable. Moreover, none of the applied algorithms uses an adaptive grid scheme. A grid structure which is not adaptive, but endogenous, is proposed by Carrol (2006) and extended by Barillas and Fernández-Villaverde (2007) and Rendahl (2007). This so called endogenous grid method defines a grid on tomorrow's variables, resulting in an endogenous grid on today's variables. Maybe most related to our paper, Gruene and Semmler (2004) propose an adaptive grid scheme for solving dynamic programming problems. However, this method is designed for value function iteration, it interpolates on rectangular elements, and is uses estimated local errors of the value function to update the grid. Along all these dimensions it is orthogonal to our algorithm. The sparse grid Smolyak 
(1963) algorithm is a well known approach to high-dimensional interpolation in economics. Krueger and Kubler (2004) use it to compute equilibria in OLG models with state spaces that have up to 30 dimensions. Certainly, this cannot be achieved in feasible time with our algorithm. However, the Smolyak algorithm requires policy functions to be smooth, which is not the case in models with occasionally binding constraints.

Section 2 presents adaptive simplicial interpolation, which consists of two components: Delaunay interpolation and an adaptive grid scheme. The example used to explain ASI is a two period exchange economy where several types of agents trade in a bond subject to ad-hoc borrowing constraints. Section 3 shows how the infinite horizon version of this economy is solved by embedding ASI in a time iteration setup. In Section 4, ASI is applied to a model where trade in a bond and a stock is subject to collateral constraints and short-selling constraints. Sections 3 and 4 examine carefully the computational performance of ASI as to the respective models. Section 5 concludes.

\section{Adaptive Simplicial Interpolation}

The main innovation of this paper is an interpolation method that is tailor made for interpolating policy functions in models with occasionally binding constraints. Section 2.1 gives a simple example of such a model: An exchange economy where heterogeneous agents trade in a one period bond subject to adhoc borrowing constraints. Section 2.2 provides a formal characterization of the problems we are considering. Section 2.3 outlines the adaptive simplicial interpolation algorithm we propose, while Sections 2.4 and 2.5 describe the two essential ingredients of the method: a simplicial interpolation technique based on Delaunay tessellation, and an adaptive grid scheme. Finally, Section 2.6 illustrates the workings of ASI with the help of the simple example from Section 2.1 . 


\subsection{Simple Example: Borrowing Constraints}

The Bond Economy

The economy is populated by $\mathrm{H}$ types of agents $\mathrm{h} \in \mathbb{H}=\{1, \ldots, H\}$ living for $T$ periods. They maximize expected time-separable lifetime utility

$$
\mathbb{E}\left[\sum_{t=1}^{T} \beta^{t} \frac{c_{t}^{1-\gamma}}{1-\gamma}\right],
$$

where $c_{t}$ denotes consumption at $t, \beta$ is the time discount factors, and $\gamma$ is the coefficient of relative risk aversion.

Uncertainty is represented by a first-order Markov process with domain $X=$ $\{1, \ldots, K\}$. Aggregate endowment of the single consumption good is given by a time invariant function $\bar{e}: X \rightarrow \mathbb{R}_{++}$, which depends on the current shock only. Similarly, agent h's individual endowment is given by $e^{h}: X \rightarrow \mathbb{R}_{++}$.

Each period, agents trade in a one period bond, which is in zero net supply. Hence, agents face the following budget constraints:

$$
c_{t}^{h}+b_{t}^{h} p_{t} \leq e_{t}^{h}+b_{t-1}^{h} \forall t=1, \ldots, T \quad \forall h \in \mathbb{H},
$$

where $b_{t}^{h}$ denotes the bond holding that agent $h$ acquires at time $t$, and $p_{t}$ denotes the respective price. Moreover, agents face an ad-hoc borrowing constraint:

$$
b_{t} \geq \underline{b} \forall t=1, \ldots, T
$$

\section{State Space}

The state of the economy at the beginning of a period is characterized by the exogenous shock and the asset distribution among agents. Because of bond market clearing, we may use the bond holdings of $H-1$ agents as the endogenous state variable:

$$
y_{t}=\left(b_{t-1}^{1}, \ldots, b_{t-1}^{H-1}\right) .
$$

Assuming that last period's constraints of all agents were satisfied, agent $h$ enters period $t$ with bond holding restricted by

$$
b_{t-1}^{h} \in[\underline{b},-\underline{b}(H-1)] .
$$


Hence, we take the endogenous state space to be

$$
Y \equiv\left\{y \in[\underline{b},-\underline{b}(H-1)]^{H-1} \mid \sum_{i=1}^{H-1} y_{i} \in[\underline{b},-\underline{b}(H-1)]\right\} .
$$

The whole state space $S$ is then given by the product of the exogenous part and the endogenous part, i.e.

$$
S=X \times Y
$$

\section{Equilibrium Conditions}

The endogenous choices and prices in period $t$ are:

$$
z_{t} \equiv\left(\left\{c_{t}^{h}, b_{t}^{h}\right\}_{h \in \mathbb{H}}, p_{t}\right)
$$

We call the collection of these endogenous variables policies, and denote the space of policies by $Z$.

The definition of competitive equilibrium is standard and given in Appendix A, where we also derive the first order necessary conditions for equilibrium. Here, we just state these conditions. Along an equilibrium path, policies satisfy market clearing on the bond market, budget constraints, Euler equations, and budget constraints and complementary slackness conditions:

$$
\begin{aligned}
& \sum_{h \in \mathbb{H}} b_{t}^{h}=0, \\
& c_{t}^{h}+b_{t}^{h} p_{t}-e_{t}^{h}-b_{t-1}^{h}=0 \quad \forall h \in \mathbb{H}, \\
& -u^{\prime}\left(c_{t}^{h}\right) p+\mu_{1}^{h}+\mathbb{E}\left[\beta u^{\prime}\left(c_{t+1}^{h}\right)\right]=0 \quad \forall h \in \mathbb{H}, \\
& 0 \leq b_{t}^{h}-\underline{b} \perp \mu_{t}^{h} \geq 0 \quad \forall h \in \mathbb{H},
\end{aligned}
$$

where $\mu^{h}$ denotes the Kuhn-Tucker multiplier on the borrowing constraint of agent $h$.

The sign $\perp$ denotes orthogonality of two vectors. Hence, for $a, b \in \mathbb{R}^{n}$ :

$$
a \perp b: \Leftrightarrow \sum_{k=1}^{n} a_{k} b_{k}=0 .
$$

If $a, b \geq 0$, then $a \perp b$ implies that for each coordinate $k=1, \ldots, n$ either $a_{k}=0$, $b_{k}=0$, or both. Hence,

$$
0 \leq a \perp b \geq 0
$$


is equivalent to

$$
\forall k=1, \ldots, n: \quad 0 \leq a_{k} \wedge 0 \leq b_{k} \wedge\left(a_{k}=0 \vee b_{k}=0\right)
$$

Two Period Version

Now consider the simplest dynamic setting: $T=2$. In this case there will be no trade in the second period, and agents simply consume all their funds:

$$
c_{2}^{h}=e_{2}^{h}+b_{1}^{h}
$$

Consequently, in period one, equilibrium conditions for given initial bond holdings $\left\{b_{0}^{h}\right\}_{h \in \mathbb{H}}$ simplify to:

$$
\begin{aligned}
& \sum_{h \in \mathbb{H}} b_{1}^{h}=0, \\
& c_{1}^{h}+b_{1}^{h} p_{1}-e_{1}^{h}-b_{0}^{h}=0 \quad \forall h \in \mathbb{H}, \\
& -u^{\prime}\left(c_{t}^{h}\right) p_{1}+\mu_{1}^{h}+\mathbb{E}\left[\beta u^{\prime}\left(e_{2}^{h}+b_{1}^{h}\right)\right]=0 \quad \forall h \in \mathbb{H}, \\
& 0 \leq b_{1}^{h}-\underline{b} \perp \mu_{1}^{h} \geq 0 \quad \forall h \in \mathbb{H} .
\end{aligned}
$$

\subsection{The General Problem}

The above problem of finding an equilibrium policy for the two period bond economy with given initial bond holdings has the following structure:

\section{Equilibrium Problem:}

Given a state $s \in S$, and functions

$\phi: S \times \mathbb{R}^{m+n} \rightarrow \mathbb{R}^{m}, \psi: S \times \mathbb{R}^{m} \rightarrow \mathbb{R}^{n}$,

find policies and multipliers $(z, \mu) \in \mathbb{R}^{m} \times \mathbb{R}^{n}$,

$$
\text { s.t. } \phi(s, z, \mu)=0, \quad 0 \leq \psi(s, z) \perp \mu \geq 0 \text {. }
$$

In the case of our example, the equations $\phi=0$ contain market clearing, budget constraints, and Euler equations. The inequalities $0 \leq \psi$ contain the borrowing constrains, and $\mu$ contains the respective Kuhn-Tucker multipliers. To solve such a problem for a given state $s$, there are many well established procedures. Either one applies solvers that accept complementarity conditions, or one transforms these conditions into equations - as explained in Appendix C - and applies standard non-linear equation solvers. 
However, things get more involved, if one is interested in how the state of the economy, $s$, maps into choices and prices, $f(s)$. Then, one faces a parametric problem, with the state of the economy, $s$, being the parameter.

\section{Parametric Equilibrium Problem:}

$$
\begin{aligned}
& \text { Given } \phi: S \times \mathbb{R}^{m+n} \rightarrow \mathbb{R}^{m}, \psi: S \times \mathbb{R}^{m} \rightarrow \mathbb{R}^{n}, \\
& \text { find } f: S \rightarrow \mathbb{R}^{m}, \quad \mu: S \rightarrow \mathbb{R}^{n}, \\
& \text { s.t. } \forall s \in S: \phi(s, f(s), \mu(s))=0, \quad 0 \leq \psi(s, f(s)) \perp \mu(s) \geq 0 .
\end{aligned}
$$

One way to compute functions $(f, \mu)$ that approximately satisfy these conditions is collocation: choose a finite grid $G \subset S$, on which the above conditions have to be satisfied precisely, i.e.

$$
\forall g \in G: \quad \phi(g, f(g), \mu(g))=0, \quad 0 \leq \psi(g, f(g)) \perp \mu(g) \geq 0 .
$$

For each point on the grid, $g \in G$, the solution $f(g)$ is determined by solving a complementarity problem. Aside from the grid $G$, collocation determines $f$ by interpolating the solutions $\{f(g)\}_{g \in G}$ found on the grid. Clearly, this does not result in a perfect fit, and more importantly, the quality of the fit depends crucially on the location of the grid points $g \in G$. In particular, if there are kinks in the function $f$, it is desirable to put grid points there, because any method that interpolates over the kink has no chance to match it exactly.

In general, $f$ is non-differentiable precisely at the points $k$ where for some $j$ both $\psi_{j}(k, f(k))=0$ and $\mu_{j}(k)=0$.

The intuition for this fact is simple: $\psi_{j}(k, f(k))=0$ means that this constraint is binding, and $\mu_{j}(k)=0$ means that the associated multiplier is zero though. Loosely speaking, the constraint is binding at one side and non-binding at the other side of the point. In general, this implies that the optimal solution is determined by different sets of equations on the two sides, resulting in different slopes of the policy function.

All in all, the above reasoning suggests that we should put interpolation nodes at points where constraints are just binding. We achieve this using the following algorithm. 


\subsection{The Algorithm}

To solve the parametric equilibrium problem presented above, we propose the following procedure.

Adaptive Simplicial Interpolation:

1. Initialization:

Start with an initial grid $G_{\text {init }}$ and solve for $\{f(g)\}_{g \in G_{\text {init }}}$ using standard numerical procedures.

2. Grid Adaptation:

Use the information $\{f(g)\}_{g \in G_{\text {init }}}$ to solve jointly for adapted grid points $G_{\text {adapt }}$ that lie directly on the kinks and for the solutions $\{f(k)\}_{k \in G_{\text {adapt }}}$ at these points. (see Section 2.5)

3. Simplicial Interpolation:

Interpolate $f$ on $G=G_{\text {init }} \cup G_{\text {adapt }}$. To interpolate on a grid with such an irregular shape, use simplicial interpolation, namely Delaunay interpolation. (See Section 2.4)

We will first explain Delaunay interpolation, as the grid adaption step builds partially on it.

\subsection{Delaunay Interpolation}

To get as much flexibility as possible in adapting the collocation grid, we need to have a method that is able to interpolate between points from any arbitrary set of scattered points. In addition, we require the method to work in arbitrary dimensions. Delaunay interpolation fulfills both criteria. This interpolation technique consists of two main steps: First, the state space is divided into simplices, which is done by Delaunay tessellation. Second, there is simplicial interpolation.

\section{Delaunay Tessellation ${ }^{2}$}

\footnotetext{
${ }^{2}$ Delaunay tessellation was first mentioned by Delaunay (1934) and is well known in areas
} 
In computational geometry, Delaunay tessellation is a well established method to cover the convex hull of an arbitrary point set with simplices. For the sake of simplicity, we explain Delaunay tessellation for the two dimensional case. In this case, the simplices are just triangles and the method is called triangulation. As one can see in Figure 2, there are various ways to triangulate a given set of points. Delaunay triangulation imposes discipline on the triangulation by satisfying the following property: inside the circumcircle of any triangle there is no point from the point set. To make sense of this requirement, note that: by definition, the vertices of a triangle lie on its circumcircle, and in a Delaunay triangulation further points might as well lie on this circumcircle but not inside. In Figure 2.4 the left hand picture shows a arbitrary set of grid points. The right hand picture shows the Delaunay Triangulation of this set.

Simpson (1978) shows that this procedure maximizes the minimum angle among all angles within the triangulation. Hence, it avoids pointed triangles. From a numerical perspective, this is a convenient property, since it implies that the information used to interpolate at a particular point stems from points that are relatively nearby. For a more extensive discussion of Delaunay Tessellation, see de Berg et al. (2008).

\section{Simplicial Interpolation}

Having the tessellation of a point set at hand, linear simplicial interpolation is straightforward: For any arbitrary point, find the simplex it is contained in. Then calculate its barycentric coordinates within this simplex. Finally, the interpolation value is given by a linear combination of the values at the corners of the simplex. The weights are given by the barycentric coordinates of the corners.

like engineering. However, up to our knowledge, it has never been used to compute equilibria in dynamic models. When it comes to economic applications, Delaunay tessellation seems to be a promising method not only for the purpose presented in this paper, namely for an adaptive grid method detecting kinks. The flexibility of Delaunay interpolation may also be used to interpolate functions on state spaces with irregular shape, or on spaces that change their shape along a time iteration procedure. Furthermore, one may also use Delaunay tessellation to adapt the grid to nonlinearities or even to jumps in the functions that are to be approximated. 

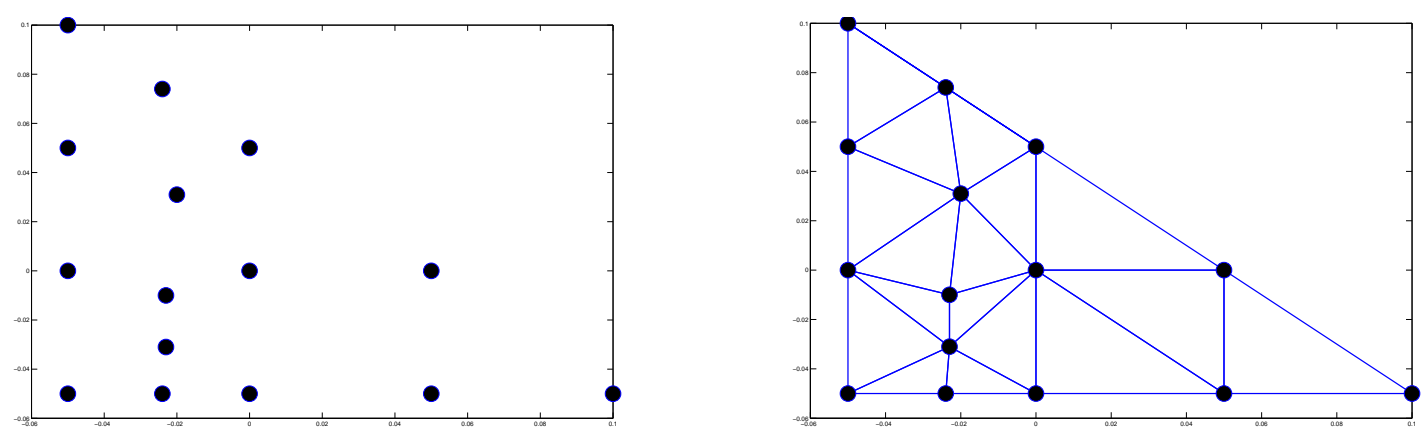

Figure 2: Set of Grid Points and Delaunay Triangulation

\subsection{An Adaptive Grid Scheme}

Let us now turn to the process of adapting the grid. Our aim is to detect kinks and place points on these kinks in order to match them precisely. In terms of the above notation, we want to determine points that lie on

$$
K=\left\{s \mid \exists j \psi_{j}(s, f(s))=0 \text { and } \mu_{j}(s)=0\right\} .
$$

Hence, we are looking for a point where a constraint holds with equality but the respective multiplier is zero, i.e. where the constraint is just binding. To determine such points we proceed as follows.

\section{How to Determine Which Edges Cross Kinks}

To determine the location of kinks, we use the solutions $\{f(g)\}$ computed on the initial grid $G_{\text {init }}$. Clearly, if $\psi_{j}(g, f(g))=0$, we know that this constraint, which we call constraint $j$, is binding at $g$. Otherwise it is not binding. Furthermore, we make use of the tessellation of the initial grid. We consider each edge of the tessellation and check whether constraint $j$ changes from being binding to non-binding from one end to the other. If this is the case, we conclude that the associated kink, which we call kink $j$, crosses this edge. In this way, we find sets of edges $\left\{\mathcal{E}_{j}\right\}$ crossing the kinks $j=1, \ldots, m$.

\section{How to Put Points Exactly on the Kink}

Given the sets of edges $\left\{\mathcal{E}_{j}\right\}$ crossing the kinks $j=1, \ldots, m$, we need to determine where exactly to put endogenous points on these edges. For each individual edge $E \in \mathcal{E}_{j}$ this is done by solving the equation system that characterizes equi- 
librium, however, not at a given point in the state space, but on the edge crossing the kink. To pin down the one point that lies on the kink, we force that both $\psi_{j}$ and $\mu_{j}$ are equal to zero. Hence, we solve jointly for the equilibrium solution and a point in the state space on which the equilibrium solution fulfills a certain requirement, namely being just binding for the considered constraint. More formally, we solve for the point $k$, policies $z$, and multipliers $\mu$ such that:

$$
\begin{aligned}
& \phi(k, z, \mu)=0, \quad 0 \leq \psi_{-j}(k, z) \perp \mu_{-j} \geq 0, \\
& \psi_{j}(k, z)=0, \quad \mu_{j}=0, \\
& k \in E
\end{aligned}
$$

By demanding $\psi_{j}(k, z)=0, \mu_{j}=0$ instead of $0 \leq \psi_{j}(k, z) \perp \mu_{j} \geq 0$ we reduce the degrees of freedom by one. But letting the state variable $k$ vary on the onedimensional object $E$, in contrast to fixing a point in the state space, increases the degrees of freedom by one. Hence, in general, there is a unique solution $(k, z, \mu)$ to this problem. This solution does not only provide the point $k$ that lies on the kink, but at the same time it provides the optimal policy at this point, namely $f(k)=z$.

In this way-for all edges $E$ in all sets $\mathcal{E}_{j}$-we compute points $k$ and policies $f(k)$. We call these points adaptive, and denote the set containing them by $G_{\text {adapt }}$. Finally, we add them to the initial points to generate the adapted grid: $G=G_{\text {init }}+G_{\text {adapt }}$. $^{3}$

\subsection{ASI at work}

Figure 3 visualizes the working principle of ASI. On the left hand side an initial grid for a particular exogenous state of the 2-period bond economy is displayed. On the $\mathrm{x}$-axis we have wealth of agent 1 , on the $\mathrm{y}$-axis wealth of agent 2 . We distributed 15 equidistant grid points over the possible combinations of wealth levels of the first two agents - remember that the wealth of agent 3 is given by market clearing. Now we solve the equilibrium problem on the initial grid.

\footnotetext{
${ }^{3}$ Instead of this fine tuned adaptation procedure, one could also use a rather mechanical update of the grid. Instead of locating the kink exactly, one could just add arbitrary points into the triangles of interest, e.g. the center point of the triangle or say 5 randomly distributed points. This is easier to compute, but comes at the cost of a less accurate result.
} 


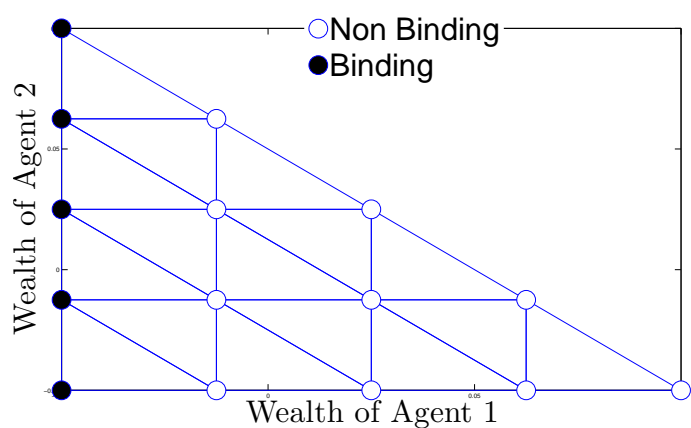

Figure 3: Initial grid (lhs) and adapted grid (rhs) using ASI in 2D

From these solutions we can infer on which edges of the triangulation constraints change from binding to non-binding. In the left picture we marked the grid points where the constraint of agent 1 is binding by dark shaded dots. The grid points where this particular constraint is not binding in the optimal solution are marked by light shaded dots. For simplicity we have chosen an example where just one constraint induces a kink into policy functions. Having detected all edges which are crossed by the kink, we now apply the second part of our adaption scheme: we solve the modified equation system that allows us to find the particular point on the edge where the constraint is just binding (e.g. where the kink crosses the edge). Doing this for all relevant edges, we end with 8 adapted points in this example, which are displayed in the right picture in Figure 3. Finally a new triangulation is computed for all grid points. Note that the new triangulation connects the kink by edges, thus kinks are matched accurately. This can also be seen in Figure 4, where the right picture shows the equilibrium bond demand function of agent 1 . The range where agent 1 is constrained by the borrowing limit is displayed by the dark shaded area. The kink induced by the inequality constraint is well approximated by the adapted points. On the right picture, we display a slice of the bond demand function of agent 1 . The dashed line illustrates how inaccurate the interpolation is at the kink, if we were to use a equidistant grid without ASI. 

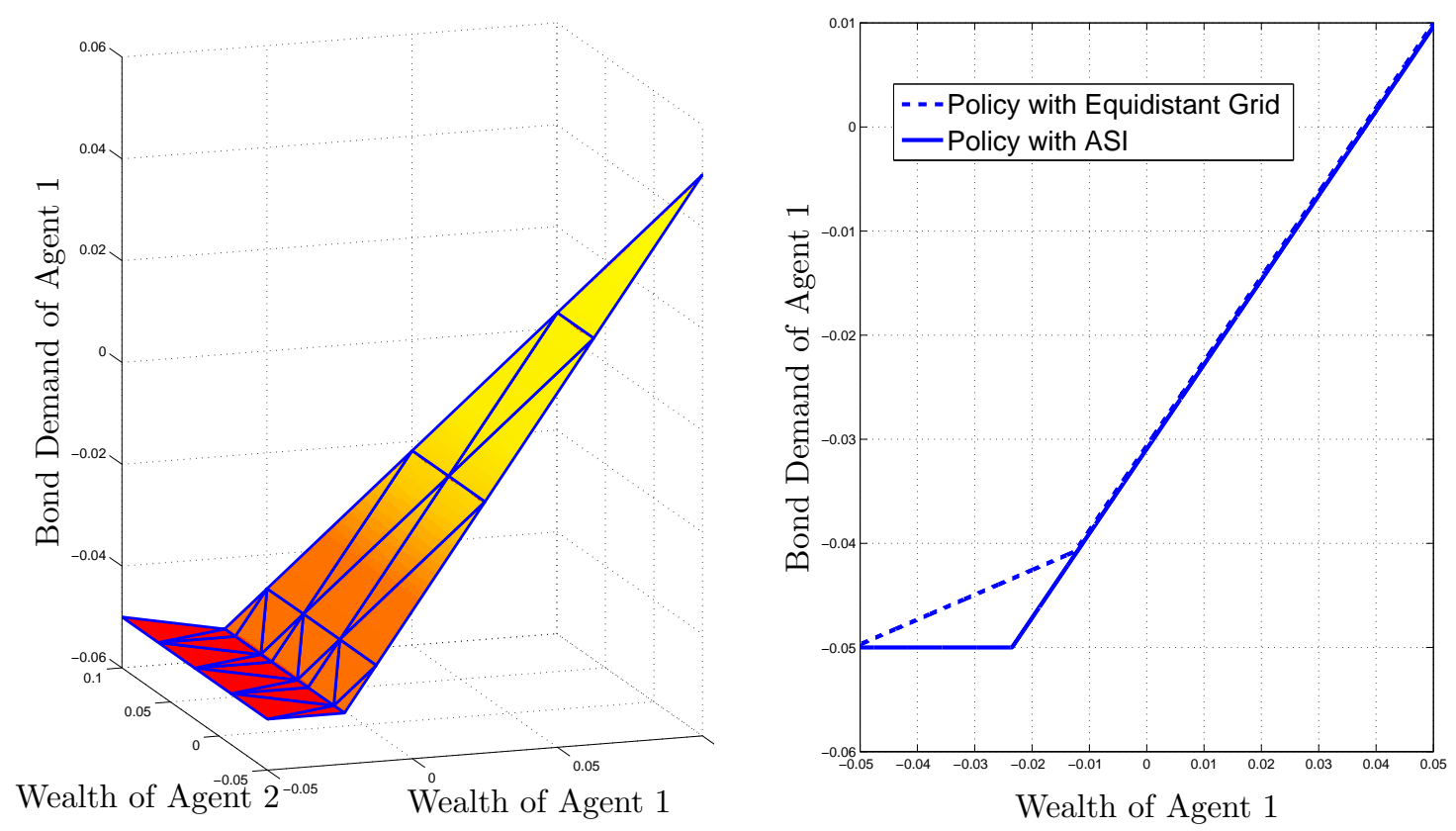

Figure 4: 2D policy with ASI (lhs) and 1D slice with and without ASI (rhs)

\section{Time Iteration with ASI}

We now consider the the infinite horizon version of the bond economy from section 2.1. Section 3.1 characterizes recursive equilibrium policies for this model. Section 3.2 shows how such policies may be computed by embedding ASI into a standard time iteration setup. Details of how we implement this algorithm are given in Section 3.3. Finally, Section 3.4 analyzes the computational performance of time iteration with ASI.

\subsection{The Infinite Horizon Bond Economy}

Consider the bond economy of Section 2.1 with $T=\infty$. We want to describe equilibrium in terms of policy functions that map the current state into current policies:

$$
f_{t}: S \rightarrow Z, f_{t}:\left(x_{t},\left(b_{t-1}^{1}, \ldots, b_{t-1}^{H-1}\right)\right) \mapsto\left(\left\{c_{t}^{h}, b_{t}^{h}\right\}_{h \in \mathbb{H}}, p_{t}\right)
$$


For the components of the policy function, we use the same notation as for their values, hence

$$
f_{t}=\left(\left\{c_{t}^{h}, b_{t}^{h}\right\}_{h \in \mathbb{H}}, p_{t}\right)
$$

For all states, these functions $\left\{f_{t}\right\}$ have to satisfy the period to period first order equilibrium conditions:

$$
\begin{aligned}
\forall s: \quad & \sum_{h \in \mathbb{H}} b^{h}(s)=0, \\
& c_{t}^{h}(s)+b_{t}^{h}(s) p_{t}(s)-e_{t}^{h}(s)-b_{t-1}^{h}(s)=0, \quad \forall h \in \mathbb{H}, \\
& -u^{\prime}\left(c_{t}^{h}(s)\right) p_{t}(s)+\mu_{t}^{h}(s)+\mathbb{E}\left[\beta u^{\prime}\left(c_{t+1}^{h}\left(s_{t+1}\right)\right)\right]=0, \quad \forall h \in \mathbb{H}, \\
& 0 \leq b_{t}^{h}(s)-\underline{b} \perp \mu_{t}^{h}(s) \geq 0, \quad \forall h \in \mathbb{H},
\end{aligned}
$$

where $s_{t+1}=\left(x_{t+1},\left(b_{t}^{1}, \ldots, b_{t}^{H-1}\right)\right)$.

A recursive equilibrium policy function of this economy is a time invariant policy function $f$ that satisfies these conditions, i.e. the sequence $\left\{f_{t}\right\}$ with $f_{t}=f \forall t$ satisfies the above conditions.

\subsection{The Algorithm}

The above period to period equilibrium conditions have the following structure:

$$
\forall s: \quad \phi\left[f^{n e x t}\right](s, f(s), \mu(s))=0, \quad 0 \leq \psi(s, f(s)) \perp \mu(s) \geq 0,
$$

where time $t$ variables have no index, and the policy in $t+1$ is denoted by $f^{\text {next }}$. The equations $\phi\left[f^{\text {next }}\right]=0$, which depend on $f^{n e x t}$, contain market clearing, budget constraints and Euler equations. Only the latter depend on $f^{n e x t}$-in this case on the consumption policies only. The inequalities $0 \leq \psi$ contain the borrowing constrains, and $\mu$ contains the respective Kuhn-Tucker multiplieres. A recursive equilibrium policy function $f$ satisfies:

$$
\forall s: \quad \phi[f](s, f(s), \mu(s))=0, \quad 0 \leq \psi(s, f(s)) \perp \mu(s) \geq 0 .
$$

The problem of finding a policy function that (approximately) satisfies this condition is very hard to address directly. In a time iteration procedure, the recursive equilibrium policy function is approximated iteratively: in each step, a 
simpler problem is solved, where next period's policy, $f^{n e x t}$, is taken as given. This brings us back to the period to period equilibrium conditions:

$$
\forall s: \quad \phi\left[f^{n e x t}\right](s, f(s), \mu(s))=0, \quad 0 \leq \psi(s, f(s)) \perp \mu(s) \geq 0 .
$$

This problem takes exactly the form of the parametric equilibrium problem discussed in Section 2.2. Hence, we may use adaptive simplicial interpolation for this essential step in the time iteration algorithm. The formal structure of the whole algorithm is given below. We deviate from a standard time iteration procedure only with regard to the interpolation procedure, which is highlighted by the inner box.

Time Iteration with Adaptive Simplicial Interpolation:

1. Select a grid $G_{\text {init }}$, an initial policy function $f^{\text {init }}$, and an error tolerance $\epsilon$. Set $f^{n e x t} \equiv f^{i n i t}$.

2. Make one time iteration step: For all $g \in G_{\text {init }}$, find $f(g)$ that solves

$$
\phi\left[f^{n e x t}\right](s, f(s), \mu(s))=0, \quad 0 \leq \psi(s, f(s)) \quad \perp \mu(s) \geq 0 .
$$

Interpolate $f$ by adaptive simplicial interpolation:

First, use the information $\{f(g)\}_{g \in G_{\text {init }}}$ to solve jointly for endogenous grid points $G_{\text {adapt }}$ that lie directly on kinks and for the optimal policy $\{f(g)\}_{g \in G_{\text {adapt }}}$ at these points.

Second, use solutions at all grid points $G=G_{\text {init }} \cup G_{\text {adapt }}$ to interpolate $f$ by simplicial interpolation.

If $\left\|f-f^{n e x t}\right\|_{\infty}<\epsilon$, go to step 3.

Else set $f^{\text {next }} \equiv f$ and repeat step 2 .

3. Set the numerical solution to the infinite horizon optimization problem: $\tilde{f}=f$.

\subsection{Implementation of the Algorithm}

To demonstrate that our algorithm works well with standard equipment, we use Matlab on an Intel Core 2 Duo $2.40 \mathrm{GHz}$ computer and standard software to 
implement our algorithm.

\section{Solving the System of Equilibrium Conditions}

To solve the complementarity problem at each grid point, one could use a solver that directly applies to complementarity problems. However, we prefer to transform the complementarity problem into a system of equations (see Appendix C) and then apply a standard non-linear equation solver, e.g. Matlab's fsolve.

\section{Adaptive Simplicial Interpolation}

Our method of choice for interpolation is Delaunay interpolation which is widely used in many areas, and hence code in several languages like $\mathrm{C}++$ or Fortran is available on the web. In Matlab, routines for computing Delaunay tessellations and simplicial interpolation come with the standard version.

\section{Time Iteration}

For the computation exercise presented below we set the error tolerance $\epsilon=10^{-5}$. We set the initial policy function $f^{\text {init }}$ such that agents consume all their wealth and the price of assets being equal to zero. Hence, $f^{\text {init }}$ corresponds to the policy function in the final period of a finite horizon economy. Generally speaking this is not an efficient starting value, but it makes the computing times of our examples comparable. To solve for the equilibrium at each grid point we use the solution from the last iteration as a starting guess. In case the solver cannot find a root we use the solution from neighboring points as new starting guesses and try again.

To decrease CPU time, we start the time iteration procedure with a relatively coarse equidistant grid, and increase the density of the grid as the error in $\left\|f-f^{n e x t}\right\|_{\infty}$ falls below $\epsilon \cdot 10$. We repeat this several times until we reach a grid of certain predefined size. In the comparison studies below, this refinement of the equidistant grid is done in exactly the same way for the adaptive grid method and the equidistant benchmark. Obviously, we do not use adaptive simplicial interpolation at each iteration step. The first step is not done until all refinements of the equidistant grid are over and the error in $\left\|f-f^{n e x t}\right\|_{\infty}$ falls below $\epsilon \cdot 10$ again. Note that kinks may change their location along the iteration, as tomorrow's policy changes. Hence, it is important to use a sufficient number of adaption steps. Further note that at each adaption step, we compute new 
adapted nodes and take out the adapted nodes from the last step as these are not needed any more.

\subsection{Computational Performence}

We evaluate the computational performance of time iteration with adaptive simplicial interpolation in two ways. We first report the accuracy of the computed equilibria using ASI for various examples. Moreover, we compare time iteration with adaptive simplicial interpolation to a standard time iteration procedure. To assess the gains from using an adaptive grid scheme, we ask the following questions: First, how do solutions on equidistant grids compare to solutions on adaptive grids, if the same number of grid points is used? And second, how many equidistant grid points are needed to match the accuracy of ASI?

\section{Measuring Accuracy}

Following Judd (1992) we evaluate the accuracy of a computed equilibrium by calculating relative errors in Euler equations (EEs), which measure the error that an agent would make in terms of his period to period consumption decision, if he used the computed policy function. The unit of measure is the relative deviation of computed (i.e. interpolated) consumption, $c_{t}^{\text {int }}$, from the one that is optimal, $c_{t}^{\text {opt }}$, given next periods interpolated consumption, $c_{t+1}^{\text {int }}$. To derive $c_{t}^{\text {opt }}$ from $c_{t+1}^{\text {int }}$ one uses an Euler equation. For instance, in the Bond economy of Section 2.1 the Euler error $E E^{h}(\cdot)$ for agent $h$ at a particular point $s$ in the state space is given by

$$
E E^{h}(s)=\left|\frac{c_{t}^{\text {opt }}}{c_{t}^{\text {int }}}-1\right|=\left|\frac{u^{\prime-1}\left(\beta \mathbb{E}_{t}\left[\frac{u^{\prime}\left(c_{t+1}^{\text {int }}\right)}{p_{\text {int }}}\right]\right)}{c_{t}^{\text {int }}(s)}-1\right|
$$

where $p_{\text {int }}$ is the interpolated price of the bond today. However, it is possible to back out $c_{t}^{\text {opt }}$ from $c_{t+1}^{\text {int }}$ only if the Kuhn-Tucker multiplier entering the Euler equation is zero, i.e. if the respective constraint is non-binding. If it is binding, we set the Euler error equal to zero instead.

Because of this problem with computing Euler errors when constraints are occasionally binding, we also report a different error measure in Appendix D. 


\section{Three Agents}

We use the following calibration: on the idiosyncratic level we assume that agents may either receive a good or a bad shock. For the case of three agents, one agent will always have the bad shock and the other two the good one. This results in three states per aggregate shock. Allowing for two aggregate shocks the exogenous part of the state space comprises six states, i.e. $|X|=6$. For the parametrization we choose values that are considered standard in the literature. We set the discount factor $\beta=0.95$ and the risk aversion parameter $\gamma=1.5$ for all agents. We values of the ratios of good to bad idiosyncratic and aggregate shocks, denoted by $\nu_{\text {idio }}$ and $\nu_{\text {agg }}$ respectively, and the persistence of idiosyncratic and aggregate shocks, $\rho_{\text {idio }}$ and $\rho_{\text {agg }}$ can be found in Table 3.1. For this calibration we compute equilibria for two values of the borrowing limit $\underline{b}$, namely $\underline{b}=0.1$ and 1, i.e. borrowing up to $10 \%$ or $100 \%$ of average individual yearly income.

\begin{tabular}{|c|c|c|c|c|c|c|}
\hline$\gamma$ & $\nu_{\text {idio }}$ & $\nu_{\text {agg }}$ & $\rho_{\text {idio }}$ & $\rho_{\text {agg }}$ & $\beta$ & $\underline{b}$ \\
\hline \hline 1.5 & 1.6 & 1.06 & 0.9 & 0.65 & 0.95 & $0.1 / 1.0$ \\
\hline
\end{tabular}

Table 3.1: Parameter values

As explained above, Euler errors are defined for a given point in the state space and a given agent. To evaluate the accuracy of computed equilibria, we calculate the Euler errors of all agents at many points in the state space. Concerning the choice of points, we make two alternative choices. First, we take random points drawn from a uniform distribution over the whole state space (EE state space). We draw 5.000 random points per exogenous shock, and compute Euler errors for all agents at these points. This results in 90.000 Euler errors per example. Second, we take the points reached along the equilibrium path, when the economy is simulated for a long 5.000 periods (EE equilibrium path). In both cases, we report both the maximum over all agents and points (max EE) as well as the average across points of the maximum across agents $(\varnothing \mathrm{EE})$. This results in four different statistics, which we all report in $\log (10)$ scale. In Table 3.2 , we provide these error measures for examples differing with respect to the borrowing limit and the number of grid points used. 
Bond Economy with Three Agents

\begin{tabular}{||c||c|c||c|c||c|c||}
\hline \hline \multicolumn{1}{||c||}{} & \multicolumn{3}{c||}{} & \multicolumn{2}{c||}{ EE state space } & \multicolumn{2}{c||}{ EE equilibrium path } \\
\hline$\underline{b}$ & points & time $(\min )$ & $\max \mathrm{EE}$ & $\varnothing \mathrm{EE}$ & $\max \mathrm{EE}$ & $\varnothing \mathrm{EE}$ \\
\hline \hline 0.1 & $\mathbf{4 0}(45)$ & $\mathbf{0 . 5}(0.4)$ & $\mathbf{- 3 . 0}(-1.2)$ & $\mathbf{- 4 . 1}(-2.1)$ & $\mathbf{- 3 . 0}(-1.2)$ & $\mathbf{- 4 . 4}(-2.1)$ \\
\hline 0.1 & $\mathbf{1 1 3}(120)$ & $\mathbf{1 . 1}(1.0)$ & $\mathbf{- 3 . 2}(-1.6)$ & $\mathbf{- 4 . 2}(-2.8)$ & $\mathbf{- 3 . 2}(-1.6)$ & $\mathbf{- 4 . 8}(-3,4)$ \\
\hline 1.0 & $\mathbf{1 8 5}(190)$ & $\mathbf{6 . 5}(4.5)$ & $\mathbf{- 2 . 1}(-1.1)$ & $\mathbf{- 3 . 1}(-2.6)$ & $\mathbf{- 2 . 2 ( - 1 . 1 )}$ & $\mathbf{- 3 . 1}(-1.8)$ \\
\hline 1.0 & $\mathbf{9 4 1}(946)$ & $\mathbf{1 3}(11)$ & $\mathbf{- 3 . 2}(-1.2)$ & $\mathbf{- 4 . 2}(-2.9)$ & $\mathbf{- 3 . 2 ( - 1 . 6 )}$ & $\mathbf{- 4 . 8}(-3.4)$ \\
\hline \hline
\end{tabular}

Table 3.2: Accuracy of adaptive grid (equidistant grid in brackets)

In all examples reported in Table 3.2, both types of Euler errors are reasonably low. Obviously, there are differences across examples regarding the level of the errors. Generally speaking, examples using more points and/or a higher borrowing limit $\underline{b}$, reducing the size of the state space in terms of consumption units, yield a lower error.

\section{Relative Performance}

In order to assess the relative performance of adaptive simplicial interpolation, we also compute equilibria on a standard equidistant grid, but still use Delaunay interpolation. The aim of this exercise is to pin down the effect of the adaptive grid. In a first comparison study, we use equidistant grids with the same number or slightly more points than the adaptive grid and assess the relative performance with respect to several error measures. In a second exercise, we want to assess by what factor one has to increase the size of equidistant grids to get the same maximum Euler error over the state space as with adaptive interpolation.

\section{Equidistant grid with same number of points}

When using the same number of points, the equidistant grid scheme is slightly faster. However, the difference is quite small, reinforcing our claim that adapting the grid takes very little time compared to overall computing time, since we jointly solve for the equilibrium, when we locate a point on the kink. More importantly, in all examples our algorithm outperforms the standard grid scheme between one and two orders of magnitude in terms of maximum error in Euler equations. This holds both for Euler errors drawn over the whole state space and 
along the equilibrium path. In the first example of Table 3.2, where we compare our results to an equidistant grid with about $5 \%$ more points, the adaptive grid yields maximum Euler errors that are about 70 times lower both on the state space and along the equilibrium path. Regarding the average Euler error, these factors are slightly lower but still substantial. We get these lower factors for average Euler errors, because the adaptive grid scheme rather targets the maximum Euler error by placing grid points on kinks, and not elsewhere in the state space. However, for two reasons the impact on average errors is also quite substantial. First, errors at the kinks are lowered dramatically, having a sizable effect on the average error. And second, even at a point located elsewhere, kinks may still play a role, because agents potentially end up near a kink tomorrow.

\section{Equidistant Grid with same Accuracy}

Second, we ask how many equidistant grid points are needed to get the same maximum Euler error as with a given adapted grid. Instead of targeting the number of grid points as above, we therefore target the maximum Euler error over the state space. For the first example with $\underline{b}=0.1$, we increase the grid size by a factor of 500. Interestingly, adaptive simplicial interpolation still outperforms the equidistant grid in terms of maximum Euler as reported in Table 3.3. Obviously, in terms of average Euler errors, taking 500 times more points has a huge impact, resulting in a lower error for the equidistant grid. For $\underline{b}=1.0$, due to memory constraints, we cannot multiply the number of nodes by 500 . We therefore increase the grid size by a factor of 120 , which yields maximum errors that are still higher than with adaptive simplicial interpolation

Bond Economy with Three Agents: Match Accuracy

\begin{tabular}{||c||c|c||c|c||c|c||}
\hline \hline \multicolumn{1}{||c||}{} & \multicolumn{2}{c||}{} & \multicolumn{2}{c||}{ EE state space } & \multicolumn{2}{c||}{ EE equilibrium path } \\
\hline$\underline{b}$ & points & time $(\min )$ & $\max \mathrm{EE}$ & $\varnothing \mathrm{EE}$ & $\max \mathrm{EE}$ & $\varnothing \mathrm{EE}$ \\
\hline \hline 0.1 & $\mathbf{4 0}(20301)$ & $\mathbf{0 . 5}(79)$ & $\mathbf{- 3 . 0}(-2.8)$ & $\mathbf{- 4 . 1}(-5.3)$ & $\mathbf{- 3 . 0}(-3.1)$ & $\mathbf{- 4 . 4}(-6.2)$ \\
\hline 1.0 & $\mathbf{1 8 5}(21945)$ & $\mathbf{6 . 5}(300)$ & $\mathbf{- 2 . 1}(-1.9)$ & $\mathbf{- 3 . 1}(-4.4)$ & $\mathbf{- 2 . 2}(-1.9)$ & $\mathbf{- 3 . 1}(-4,6)$ \\
\hline \hline
\end{tabular}

Table 3.3: Accuracy of adaptive grid (equidistant grid in brackets)

To illustrate our findings we have visualized the second example of Table 3.2, 

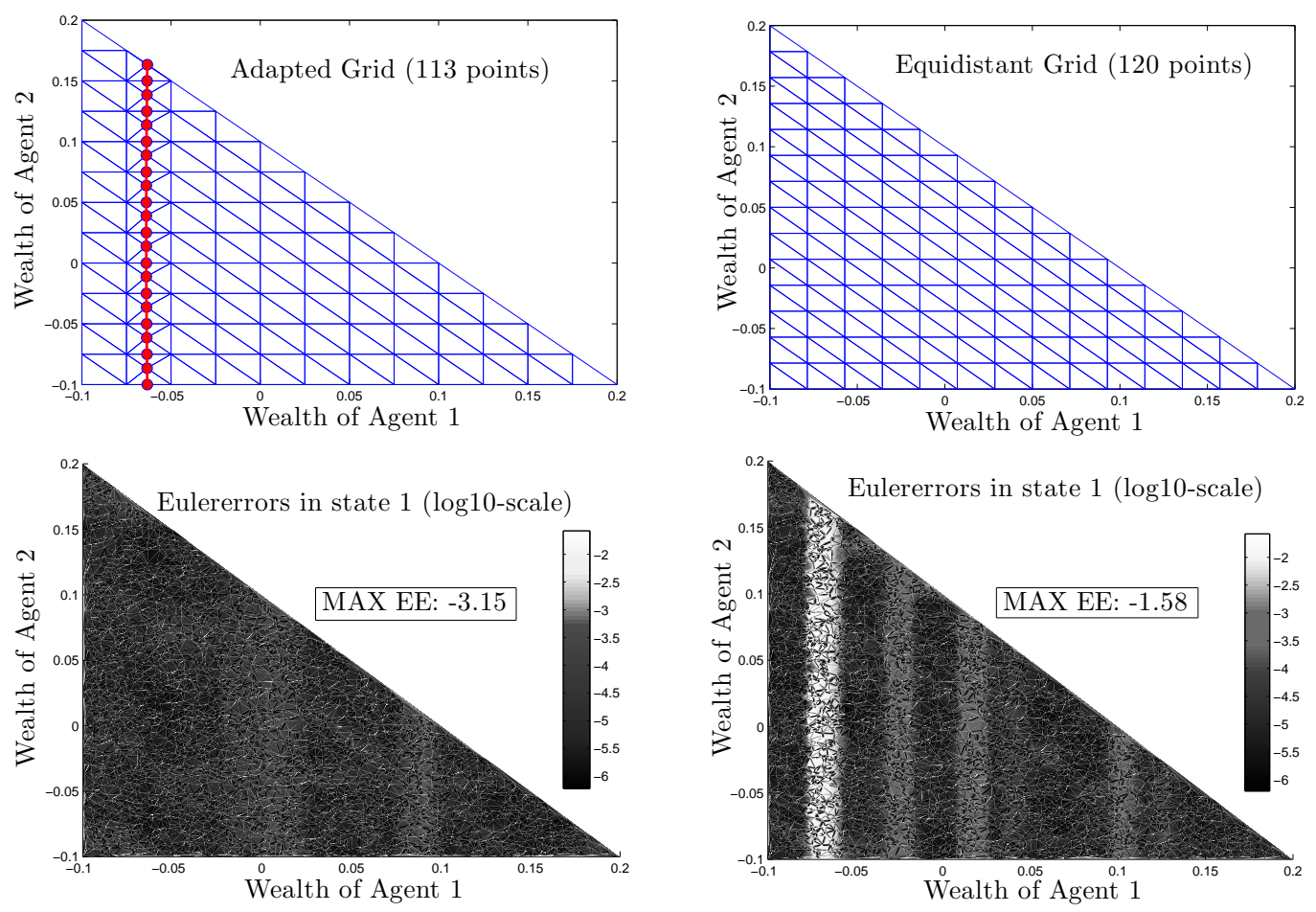

Figure 5: Grid and EEs for adapted (lhs) and equidistant (rhs) case

where the adaptive grid used 113 points. The upper left picture in Figure 5 shows the adapted grid, where again the dots mark the adapted points lying on the kinks. In this example, there is one kink, which runs vertically through the state space. This kink is associated with the borrowing constraint of the first agent, who receives a bad idiosyncratic shock, forcing his borrowing constraint to be binding in a large part of the state space. The lower two pictures display Euler errors for the two equilibria computed with adaptive simplicial interpolation and with a standard equidistant grid. The size of the errors in $\log (10)$-scale is reflected by the levels of gray, where lower errors are pictured darker. In the case of the equidistant grid, the white shaded areas representing the highest Euler errors lie around the kinks. Comparing the two lower pictures, one can see that the high Euler errors vanish completely when the adaptive grid is used.

\section{Four Agents}

In case of the Bond economy we also compute equilibria for four agents imply- 


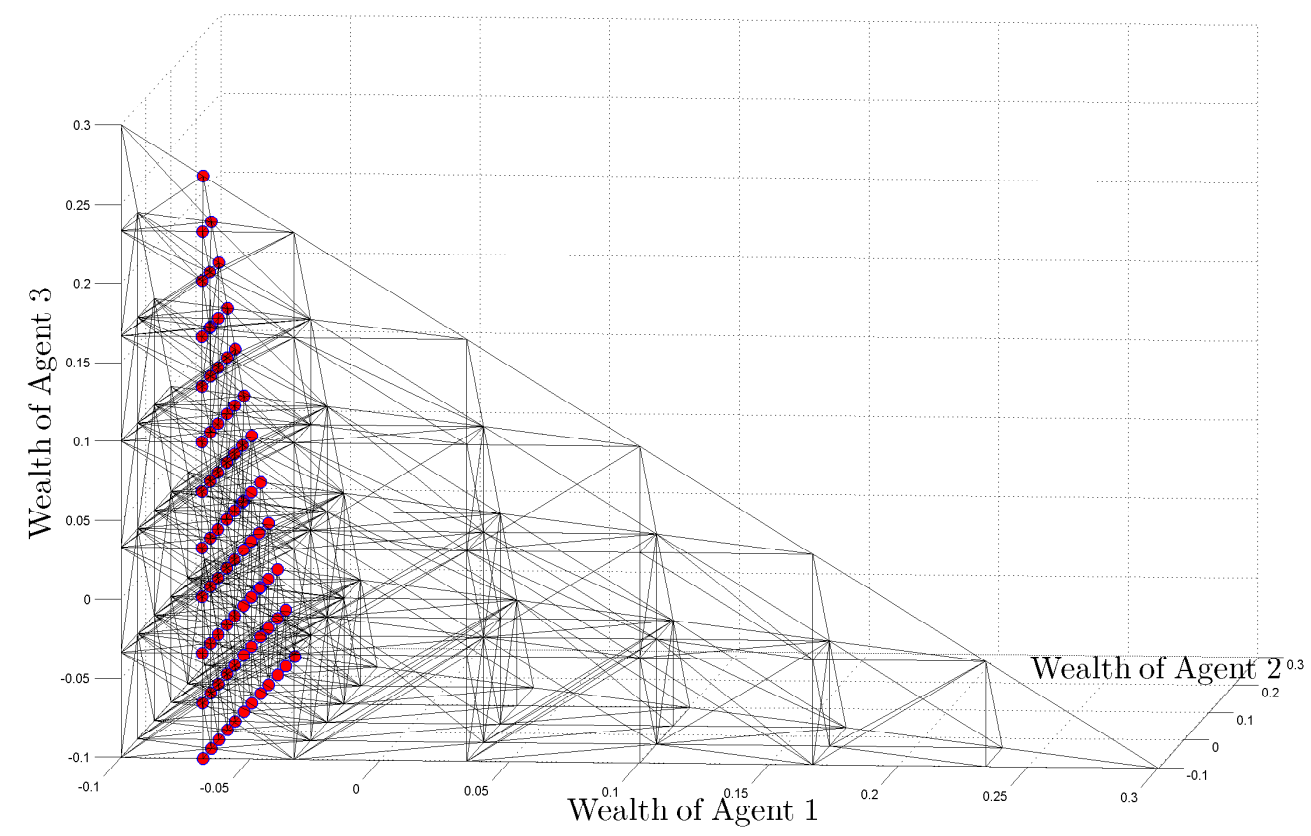

Figure 6: Adapted Grid with Three Continuous State Variables

ing three continuous state variables. We stick to the above calibration with one exceptions: we now assume that there is always one agent receiving the bad idiosyncratic shock and 3 agents receiving the good one. Therefore the exogenous part of the state space consists of eight states, i.e $|X|=8$. In the case of a three-dimensional state space kinks become two dimensional objects, as the right picture in Figure 6 indicates: the grid is adapted to one kink which lies approximately orthogonal to the horizontal axis. In Table 3.4 we provide accuracy results, showing that the algorithm is also able to deal with three dimensional state spaces.

\section{Bond Economy with Four Agents}

\begin{tabular}{||c||c|c||c|c||c|c||}
\hline \hline \multicolumn{1}{||c||}{} & \multicolumn{3}{c||}{} & \multicolumn{2}{c||}{ EE state space } & \multicolumn{2}{c||}{ EE equilibrium path } \\
\hline$\underline{b}$ & points & time $(\min )$ & $\max \mathrm{EE}$ & $\varnothing \mathrm{EE}$ & $\max \mathrm{EE}$ & $\varnothing \mathrm{EE}$ \\
\hline \hline 0.1 & $\mathbf{1 1 2}(120)$ & $\mathbf{4 . 5}(4)$ & $\mathbf{- 2 . 7}(-1.3)$ & $\mathbf{- 3 . 3}(-2.0)$ & $\mathbf{- 2 . 7}(-1.3)$ & $\mathbf{- 3 . 9}(-1.7)$ \\
\hline 1.0 & $\mathbf{9 1 4}(969)$ & $\mathbf{6 0}(51)$ & $\mathbf{- 1 . 7}(-1.1)$ & $\mathbf{- 2 . 6}(-2.4)$ & $\mathbf{- 1 . 8}(-1.1)$ & $\mathbf{- 2 . 6}(-3.9)$ \\
\hline \hline
\end{tabular}

Table 3.4: Accuracy of adaptive grid (equidistant grid in brackets) 
Bond Economy with Four Agents: Match Accuracy

\begin{tabular}{||c||c|c||c|c||c|c||}
\hline \hline \multicolumn{1}{||c||}{} & \multicolumn{2}{c||}{} & \multicolumn{2}{c||}{ EE state space } & \multicolumn{2}{c||}{ EE equilibrium path } \\
\hline$\underline{b}$ & points & time $(\min )$ & $\max \mathrm{EE}$ & $\varnothing \mathrm{EE}$ & $\max \mathrm{EE}$ & $\varnothing \mathrm{EE}$ \\
\hline \hline 0.1 & $\mathbf{1 1 2}(20825)$ & $\mathbf{4 . 5}(895)$ & $\mathbf{- 2 . 7}(-2.0)$ & $\mathbf{- 3 . 3}(-3.6)$ & $\mathbf{- 2 . 7}(-2.1)$ & $\mathbf{- 3 . 9}(-4.0)$ \\
\hline 1.0 & $\mathbf{9 1 4}(20825)$ & $\mathbf{9 0}(3655)$ & $\mathbf{- 1 . 7}(-1.1)$ & $\mathbf{- 2 . 6}(-3.0)$ & $\mathbf{- 1 . 8}(-1.1)$ & $\mathbf{- 2 . 6}(-1.9)$ \\
\hline \hline
\end{tabular}

Table 3.5: Accuracy of adaptive grid (equidistant grid in brackets)

Relative Performance

As in the case of three agents, we compare the results from ASI to equilibria com-

puted on equidistant grids. Concerning the comparison using the same amount of grid points, we again find that ASI outperforms equidistant grid points by far. Trying to match the maximum Euler Error from the ASI example, we increase the amount of grid points by a factor of 200 for $\underline{b}=0.1$ and 20 for $\underline{b}=1.0$. For both cases we find that the maximum Euler Error on the equidistant grid is still far higher, mirroring the result from the model with three agents.

\section{Extension: Endogenous Collateral Constraints}

\subsection{The Bond and Stock Economy}

Setup

We extend the bond economy of Section 2.1 by introducing a Lucas tree-type stock which is in unit net supply. It pays out a fixed fraction $\alpha$ of aggregate endowments each period, i.e. stock holders receive dividends $d(x)=\alpha \cdot \bar{e}(x)$ per unit of the stock. Hence, aggregate endowment is given by the sum of individual endowments and dividends, i.e.

$$
\bar{e}(x)=\sum_{h \in \mathbb{H}} e^{h}(x)+d(x) \forall x \in X .
$$

The Lucas tree is traded each period after dividends are payed. Each agent $\mathrm{h}$ buys $l^{h}$ shares of the stock at a price $q$. Hence, agents face the following budget constraints:

$$
c_{t}^{h}+b_{t}^{h} p_{t}+l_{t}^{h} q_{t} \leq e_{t}^{h}+b_{t-1}^{h}+l_{t-1}^{h}\left(q_{t}+d_{t}\right) \quad \forall t=1, \ldots, T \quad \forall h \in \mathbb{H},
$$


Moreover, trade in the bond and the stock is subject to constraints. First, we impose a short-selling constraint on the stock, i.e.

$$
l_{t}^{h} \geq 0 \forall t=1, \ldots, T \forall h \in \mathbb{H} \text {. }
$$

More importantly, agents face endogenous collateral constraints. These constraints arise through a bankruptcy law which makes it possible to seize an agents' financial wealth but not his income. To put it differently, all future income is exempted. As there is no further punishment for default, an agent will default on his asset position, if and only if his portfolio has a negative value. As this behavior is anticipated - and we assume that default premia may not be charged $^{4}$ - no agent will be allowed to acquire such a portfolio. This imposes the following trading constraints:

$$
\min _{x_{t+1} \in X}\left\{l_{t}^{h}\left(q\left(s_{t+1}\right)+d\left(x_{t+1}\right)\right)+b_{t}^{h}\right\} \geq 0, \forall t=1, \ldots, T \quad \forall h \in \mathbb{H},
$$

where $s_{t+1}=\left(x_{t+1}, y_{t+1}\right)$, and the endogenous part of the state, $y_{t+1}$, is still to be specified.

\section{State Space}

With the above collateral constraint, financial wealth,

$$
w_{t}^{h} \equiv l_{t-1}^{h}\left(q\left(s_{t}\right)+d\left(x_{t}\right)\right)+b_{t-1}^{h},
$$

cannot go below zero. Hence, the fraction of total financial wealth that an agent holds,

$$
y^{h}=\frac{w^{h}}{\sum_{j \in \mathbb{H}} w^{j}}
$$

is bounded between zero and one. By market clearing, we may use the fractions of financial wealth of the first $H-1$ agents as the endogenous state space:

$$
y=\left(y^{1}, \ldots, y^{H-1}\right) \in Y \equiv\left\{y \in \mathbb{R}_{+}^{H-1} \mid \sum_{i=1}^{H-1} y^{i} \leq 1\right\} \subset \mathbb{R}_{+}^{H-1} .
$$

\footnotetext{
${ }^{4}$ The reason may be that a bankruptcy procedure is extremely costly in spite of the fact that creditors are committed to use this option.
} 
Finally, we define the whole state space $S$ as the product of the exogenous part and the endogenous part, i.e.

$$
S=X \times Y
$$

With this definition of the state space, reconsider the collateral constraint above, and note that: Todays choice of any agent, through its impact on tomorrows state, influences tomorrows price of the stock, and hence today's collateral constraint of agent $h$. In this sense, the collateral constraint is endogenous, which complicates the model considerably.

\section{Equilibrium Conditions}

The endogenous choices and prices in period $t$ are

$$
z_{t} \equiv\left(\left(c_{t}^{h}, b_{t}^{h}, l_{t}^{h}\right)_{h \in \mathbb{H}}, p_{t}, q_{t}\right) .
$$

In Appendix B we define competitive equilibrium and derive the first order equilibrium conditions of this model. Along an equilibrium path, policies have to satisfy market clearing on both asset markets, budget constraints, Euler equations for both assets, and complementary slackness conditions for both kinds of multipliers:

$$
\begin{aligned}
& \sum_{h \in \mathbb{H}} b_{t}^{h}=0, \quad \sum_{h \in \mathbb{H}} l_{t}^{h}=1, \\
& c_{t}^{h}+b_{t}^{h} p_{t}+l_{t}^{h} q_{t}-e_{t}^{h}-b_{t-1}^{h}-l_{t-1}^{h}\left(q_{t}+d_{t}\right)=0, \quad \forall h \in \mathbb{H}, \\
& -u^{\prime}\left(c_{t}^{h}\right) p_{t}+\mu^{h}+\mathbb{E}\left[\beta u^{\prime}\left(c_{t+1}^{h}\right)\right]=0, \quad \forall h \in \mathbb{H}, \\
& -u^{\prime}\left(c_{t}^{h}\right) q_{t}+\mu^{h} \min _{x_{t+1} \in X}\left\{q\left(s_{t+1}\right)+d\left(x_{t+1}\right)\right\}+\nu_{t}^{h}+\mathbb{E}\left[\beta u^{\prime}\left(c_{t+1}^{h}\right)\left(q_{t+1}+d_{t+1}\right)\right]=0, \quad \forall h \in \mathbb{H}, \\
& 0 \leq \min _{x_{t+1} \in X}\left\{l_{t}^{h}\left(q\left(s_{t+1}\right)+d\left(x_{t+1}\right)\right)+b_{t}^{h}\right\} \quad \perp \mu_{t}^{h} \geq 0, \quad \forall h \in \mathbb{H}, \\
& 0 \leq l_{t}^{h} \perp \nu_{t}^{h} \geq 0, \quad \forall h \in \mathbb{H},
\end{aligned}
$$

where $\mu^{h}$ and $\nu^{h}$ denote the Kuhn-Tucker multipliers on the collateral and the short-selling constraint of agent $h$.

\subsection{Computational Performance}

Before we look at errors in Euler equation, we first want to discuss how the short selling and collateral constraints induce kinks that run in non-standard 


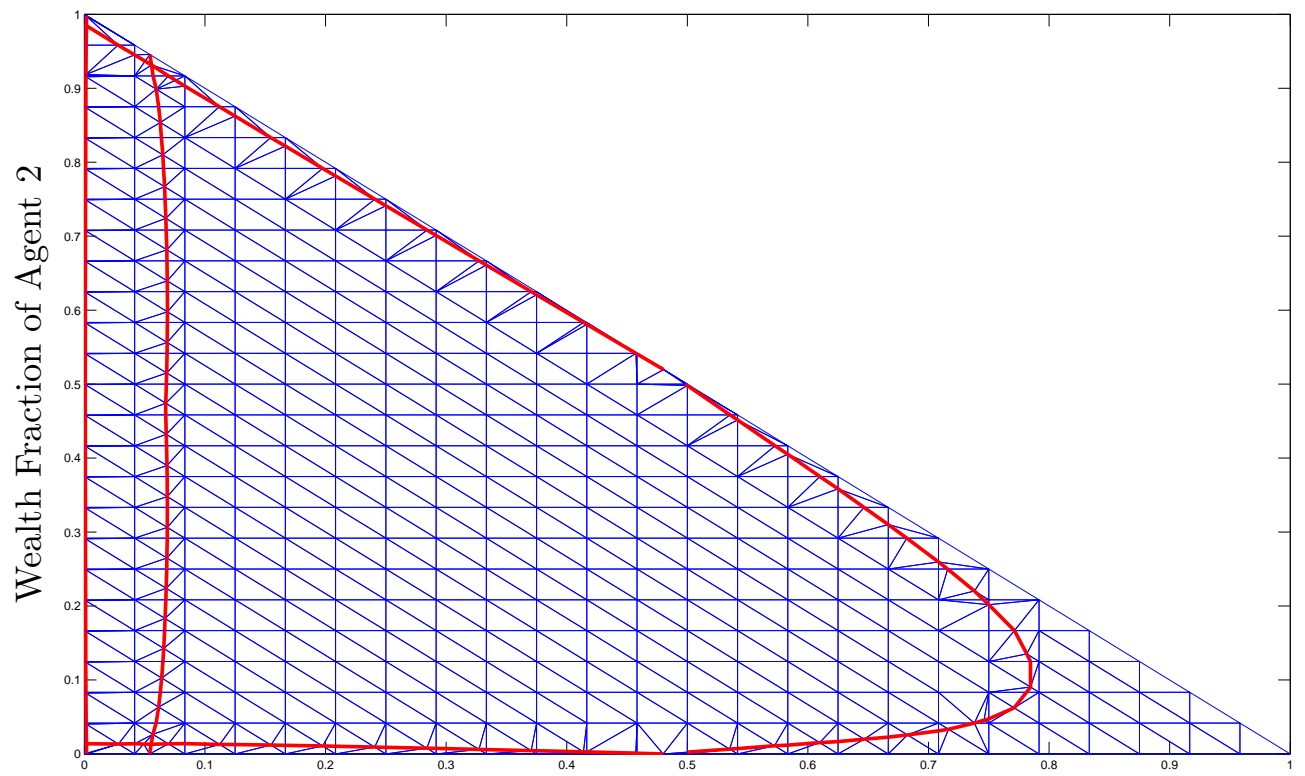

Wealth Fraction of Agent 1

Figure 7: Bond and Stock Economy: Grid with 5 Kinks

ways through the state space. Consider a particular example where we use a dividend parameter $\alpha=0.1$ and look at an exogenous state, where the first agent is hit by a bad idiosyncratic shock. Figure 7 shows the adapted grid where altogether five kinks have been identified. We highlight the edges that connect adapted points to clearly visualize the kinks. Two constraints, the one very close to the y-axis and the curved one to the very right, are induced by the short selling constraint of the first agent. Furthermore, each of the collateral constraints induces one kink, where the kink from the first agent's constraint is the one that runs parallel to the y-axis approximately at 0.08 fraction of wealth of agent 1. In Figure 8 an equilibrium stock demand function of agent 1 for the considered exogenous state is displayed: the left picture shows the stock demand over the whole state space, whereas the picture on the right hand side displays a slice of this function, at 0.1 wealth fraction of agent 2 . The distinct peak at 0.08 wealth fraction of agent 1 corresponds to the kink induced by his collateral constraint. At higher levels of wealth his demand for the stock goes down until the short selling constraint becomes binding again.

Performance of ASI 

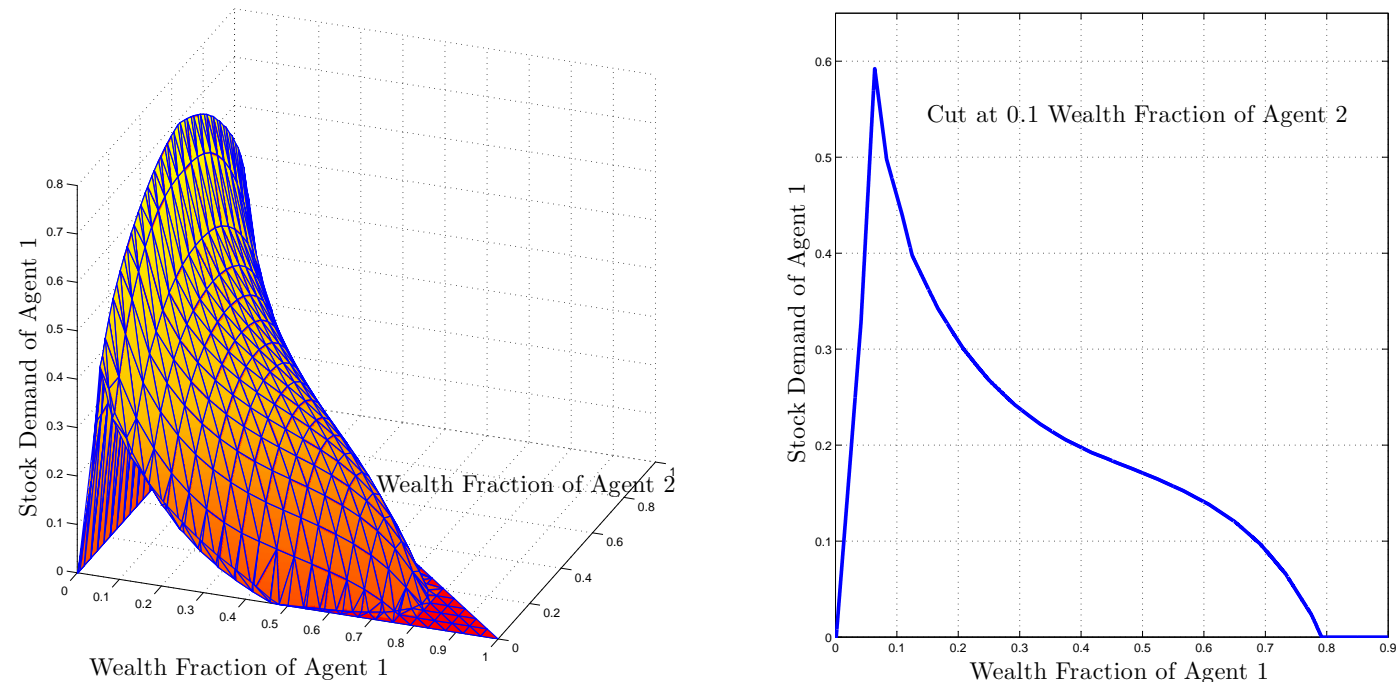

Figure 8: Bond and Stock Economy: Stock Demand

We again evaluate the performance of our algorithm by computing relative errors in Euler equations. In Table 4.1, we show results for equilbria computed with ASI. Obviously, as the figures above suggest, more points are needed than in the bond model to bring Euler errors down to reasonable values. Comparing the results from ASI with results on equidistant grids, we again see that for the same number of grid points, ASI outperforms equidistant grids by approximately one order of magnitude in terms of maximum Euler Error. Again, we ask the question how many points are needed to match the accuracy of ASI. Increasing the number of points by a factor of 20 yields almost the same maximum Euler Error, as the examples in Table 4.2 show. This factor is still substantial, however, not as high as for the Bond model. The reason are non-linearities that occur away from the kink, as can be seen in Figure 8. We have developed an adaption scheme that is tailor-made to adapt the grid to non-linearities, improving further the relative performance of our algorithm. However, as this is not the focus of this paper, we are not going to elaborate more on this. 
Bond and Stock Economy

\begin{tabular}{||c||c|c||c|c||c|c||}
\hline \hline & \multicolumn{2}{|c||}{} & \multicolumn{2}{c||}{ EE state space } & \multicolumn{2}{c||}{ EE equilibrium path } \\
\hline & points & time $(\min )$ & $\max \mathrm{EE}$ & $\varnothing \mathrm{EE}$ & $\max \mathrm{EE}$ & $\varnothing \mathrm{EE}$ \\
\hline \hline & $\mathbf{1 2 3 5}(1250)$ & $\mathbf{3 1 0}(260)$ & $\mathbf{- 2 . 5}(-1.4)$ & $\mathbf{- 3 . 8}(-3.2)$ & $\mathbf{- 3 . 1}(-1.6)$ & $\mathbf{- 4 . 1}(-3.5)$ \\
\hline \hline
\end{tabular}

Table 4.1: Accuracy of adaptive grid (equidistant grid in brackets)

Bond and Stock Economy: Match of Accuracy

\begin{tabular}{|c|c|c|c|c|c|}
\hline & & \multicolumn{2}{|c|}{ EE state space } & \multicolumn{2}{|c|}{ EE equilibrium path } \\
\hline points & time $(\min )$ & $\max \mathrm{EE}$ & $\varnothing \mathrm{EE}$ & $\max \mathrm{EE}$ & $\varnothing \mathrm{EE}$ \\
\hline $\mathbf{1 2 3 5}(25400)$ & $310(4500)$ & $\mathbf{- 2 . 5}(-2.4)$ & $\mathbf{- 3 . 8}(-4.0)$ & $\mathbf{- 3 . 1}(-2.6)$ & $-4.1(-4.2)$ \\
\hline
\end{tabular}

Table 4.2: Accuracy of adaptive grid (equidistant grid in brackets)

\section{Conclusion}

This paper presents a new algorithm to compute equilibria in dynamic models with occasionally binding constraints. To address directly the problem of kinks in such models, we develop a new interpolation technique based on adaptive grids and simplicial interpolation. We show that Adaptive Simplicial Interpolation accurately computes equilibria in dynamic models with several continuous state variables, up to two assets and many inequality constraints. Comparison studies show that our method outperforms standard equidistant grid techniques by about two order of magnitude in terms of maximum errors in Euler equations.

\section{References}

Barillas, F. and J. Fernández-Villaverde (2007). A generalization of the endogenous grid method. Journal of Economic Dynamics and Control 31(8), 2698-2712.

Carrol, C. D. (2006). The method of endogenous gridpoints for solving dynamic stochastic optimization problems. Economics Letters 91(3), 312-320.

Christiano, L. J. and J. D. Fisher (2000). Algorithms for solving dynamic models 
with occasionally binding constraints. Journal of Economic Dynamics and Control 24(8), 1179-1232.

de Berg, M., O. Cheong, M. van Kreveld, and M. Overmars (2008). Computational Geometry: Algorithms and Applications. Springer.

Delaunay, B. (1934). Sur la sphère vide. Bulletion of Academy of Sciences of the USSR 7 6, 793-800.

den Haan, W. J. (2001). The importance of the number of different agents in a heterogeneous asset-pricing model. Journal of Economic Dynamics and Control 25(5), 721-746.

Garcia, C. and W. Zangwill (1981). Pathway to Solutions, Fixed Points and Equilibria. Prentice Hall, Englewood Cliffs.

Gruene, L. and W. Semmler (2004). Using dynamic programming with adaptive grid scheme for optimal control problems in economics. Journal of Economic Dynamics and Control 28, 2427-2456.

Heaton, J. and D. Lucas (1996). Evaluating the effects of incomplete markets on risk sharing and asset pricing. Journal of Political Economy 104, 443-487.

Judd, K. (1992). Projection methods for solving aggregate growth models. Journal of Economic Theory 58, 410-452.

Judd, K. (1998). Numerical Methods in Economics. MIT Press, Cambridge.

Judd, K., F. Kubler, and K. Schmedders (2003). Computational methods for dynamic equilibria with heterogeneous agents. In M. Dewatripont, L. P. Hansen, and S. Turnovsky (Eds.), Advances in Economics and Econometrics, pp. 243290. Cambridge University Press.

Krueger, D. and F. Kubler (2004). Computing equilibrium in olg models with stochastic production. Journal of Economic Dynamics and Control 28(7), $1411-1436$.

Kubler, F. and K. Schmedders (2003). Stationary equilibria in asset pricingmodels with incomplete markets and collateral. Econometrica 71, 1767-1793. 
Rendahl, P. (2007). Inequality constraints in recursive economics. Mimeo, European University Institute.

Simpson, R. (1978). Locally equiangular triangulations. The Computer Journal 21, 243-245.

Smolyak, S. (1963). Quadrature and interpolation formulas for tensor products of certain classes of functions. Soviet Mathematics Doklady 4, 240-243. 


\section{A Details Bond Economy}

$((($ tbd: similar to Appendix B $)))$

\section{B Details Bond and Stock Economy}

In this appendix, we define competitive equilibrium and derive first order equilibrium conditions for the economy presented in Section 4. For this purpose, some additional notation is needed. We denote the shock at time $\mathrm{t}$ by $x_{t}$, but the history of shocks that occurred up to period t by $x^{t}$. The set of histories up to period $\mathrm{t}$ is denoted by $X^{t}$, and the set of all possible histories by $\mathbb{X} \equiv \bigcup_{t=1}^{\infty} X^{t}$. For $x^{t+1}$ being a possible successor of $x^{t}$ we write $x^{t+1} \geq x^{t}$. Finally, the probability of history $x^{t}$ is denoted by $\pi\left(x^{t}\right)$.

Competitive Equilibrium

A competitive equilibrium for an economy with agents' initial portfolios

$$
\left(b_{0}^{h}, l_{0}^{h}\right)_{h \in \mathbb{H}}
$$

is a collection

$$
\left\{z\left(x^{t}\right)\right\}_{x^{t} \in \mathbb{X}} \equiv\left(\left(c^{h}\left(x^{t}\right), b^{h}\left(x^{t}\right), l^{h}\left(x^{t}\right)\right)_{h \in \mathbb{H}}, p\left(x^{t}\right), q\left(x^{t}\right)\right)_{x^{t} \in \mathbb{X}}
$$

of consumption allocations, bond and stock holdings, and prices that satisfy the following conditions:

1. Markets clear ${ }^{5}$ :

$$
\sum_{h \in \mathbb{H}} b^{h}\left(x^{t}\right)=0, \quad \sum_{h \in \mathbb{H}} l^{h}\left(x^{t}\right)=1 \quad \forall x^{t} \in \mathbb{X} .
$$

2. Given prices $\left(p\left(x^{t}\right), q\left(x^{t}\right)\right)_{x^{t} \in \mathbb{X}}$, each agent chooses

$$
\left.\left(c^{h}\left(x^{t}\right), b^{h}\left(x^{t}\right), l^{h}\left(x^{t}\right)\right)\right)_{x^{t} \in \mathbb{X}}
$$

\footnotetext{
${ }^{5}$ By Walras' Law market clearing in the asset markets implies market clearing in the consumption goods market.
} 
to maximize lifetime utility such that $\forall x^{t} \in \mathbb{X}$ the following constraints hold:

$$
\begin{aligned}
\text { budget constraint } & c^{h}\left(x^{t}\right)+b^{h}\left(x^{t}\right) p\left(x^{t}\right)+l^{h}\left(x^{t}\right) q\left(x^{t}\right) \leq \\
& e^{h}\left(x^{t}\right)+b^{h}\left(x^{t-1}\right)+l^{h}\left(x^{t-1}\right)\left(q_{t}\left(x^{t}\right)+d_{t}\left(x^{t}\right)\right), \\
\text { short selling constraint } & l^{h}\left(x^{t}\right) \geq 0 \text { and } \\
\text { collateral constraints } & \min _{x^{t+1} \geq x^{t}}\left\{l^{h}\left(x^{t}\right)\left(q\left(x^{t+1}\right)+d\left(x^{t+1}\right)\right)+b^{h}\left(x^{t}\right)\right\} \geq 0 .
\end{aligned}
$$

\section{First Order Equilibrium Conditions}

Each individual agent faces the following optimization problem:

$$
\begin{aligned}
& \max _{\left(c\left(x^{t}\right), b\left(x^{t}\right), l\left(x^{t}\right)\right)_{x^{t} \in \mathbb{X}}} \mathbb{E}\left[\beta^{t} u\left(c\left(x^{t}\right)\right)\right] \\
& \text { s.t. } \forall x^{t} \in \mathbb{X}:
\end{aligned}
$$

budget constraint

$$
\begin{aligned}
& c^{h}\left(x^{t}\right)+b^{h}\left(x^{t}\right) p\left(x^{t}\right)+l^{h}\left(x^{t}\right) q\left(x^{t}\right) \leq \\
& e^{h}\left(x^{t}\right)+b^{h}\left(x^{t-1}\right)+l^{h}\left(x^{t-1}\right)\left(q_{t}\left(x^{t}\right)+d_{t}\left(x^{t}\right)\right),
\end{aligned}
$$

short selling constraint $\quad l^{h}\left(x^{t}\right) \geq 0$ and

$$
\text { collateral constraints } \min _{x^{t+1} \geq x^{t}}\left\{l^{h}\left(x^{t}\right)\left(q\left(x^{t+1}\right)+d\left(x^{t+1}\right)\right)+b^{h}\left(x^{t}\right)\right\} \geq 0 .
$$

Denote the multiplier associated with these constraints by $\lambda\left(x^{t}\right), \nu\left(x^{t}\right)$, and $\mu\left(x^{t}\right)$. Differentiating the Lagrangian gives

$$
\begin{array}{rll}
c\left(x^{t}\right): & \pi\left(x^{t}\right) \beta^{t} u^{\prime}\left(c\left(x^{t}\right)\right)-\lambda\left(x^{t}\right)=0 \\
c\left(x^{t+1}\right): & \pi\left(x^{t+1}\right) \beta^{t+1} u^{\prime}\left(c\left(x^{t+1}\right)\right)-\lambda\left(x^{t+1}\right)=0 \\
b\left(x^{t}\right): & -\lambda\left(x^{t}\right) p\left(x^{t}\right)+\mu\left(x^{t}\right)+\sum_{x^{t+1} \geq x^{t}}\left(\lambda\left(x^{t+1}\right)\right)=0 \\
l\left(x^{t}\right): & \nu\left(x^{t}\right)-\lambda\left(x^{t}\right) q\left(x^{t}\right)+\mu\left(x^{t}\right) \min _{x^{t+1} \geq x^{t}}\left\{q\left(x^{t+1}\right)+d\left(x^{t+1}\right)\right\} \\
& +\sum_{x^{t+1} \geq x^{t}}\left(\lambda\left(x^{t+1}\right)\right)\left(q\left(x^{t+1}\right)+d\left(x^{t+1}\right)\right)=0 .
\end{array}
$$

Substituting the first two FOCs into the other three, we get the following Euler 
equations for the bond and the stock:

$$
\begin{aligned}
& -u^{\prime}\left(c\left(x^{t}\right)\right) p\left(x^{t}\right)+\mu\left(x^{t}\right)+\sum_{x^{t}+\geq x^{t}}\left(\beta \pi\left(x^{t+1} \mid x^{t}\right) u^{\prime}\left(c\left(x^{t+1}\right)\right)\right)=0, \\
& \nu\left(x^{t}\right)-u^{\prime}\left(c\left(x^{t}\right)\right) q\left(x^{t}\right)+\mu\left(x^{t}\right) \min _{x^{t+1} \geq x^{t}}\left\{q\left(x^{t+1}\right)+d\left(x^{t+1}\right)\right\} \\
& +\sum_{x^{t+1} \geq x^{t}}\left(\beta \pi\left(x^{t+1} \mid x^{t}\right) u^{\prime}\left(c\left(x^{t+1}\right)\right)\right)\left(q\left(x^{t+1}\right)+d\left(x^{t+1}\right)\right)=0 .
\end{aligned}
$$

In addition, the Kuhn-Tucker FOCs include the following complementarity conditions:

$$
\begin{aligned}
& 0 \leq \mu\left(x^{t}\right) \perp \min _{x^{t+1} \geq x^{t}}\left\{l^{h}\left(x^{t}\right)\left(q\left(x^{t+1}\right)+d\left(x^{t+1}\right)\right)+b^{h}\left(x^{t}\right)\right\} \geq 0 \\
& 0 \leq \nu\left(x^{t}\right) \perp l\left(x^{t}\right) \geq 0 .
\end{aligned}
$$

Combined with market clearing conditions and budget constraints, these are the equilibrium conditions stated in Section 4.

\section{Transforming Complementarities into Equa- tions}

At the initial gridpoints, ASI solves the follwing complementarity problem:

Given a state $s \in S$, and functions

$\phi: S \times \mathbb{R}^{m+n} \rightarrow \mathbb{R}^{m}, \psi: S \times \mathbb{R}^{m} \rightarrow \mathbb{R}^{n}$,

find policies and multipliers $(z, \mu) \in \mathbb{R}^{m} \times \mathbb{R}^{n}$,

s.t. $\phi(s, z, \mu)=0, \quad 0 \leq \psi(s, z) \perp \mu \geq 0$.

Inspired by Garcia and Zangwill (1981), we transform this complementarity problem into a system of equations, to be able to apply a standard non-linear equation solver. Key to the transformation are the following definitions:

$$
\alpha \equiv \begin{cases}\mu & \text { for } \quad \mu \geq 0, \quad \psi(s, z)=0 \\ -\psi(s, z) & \text { for } \quad \mu=0, \quad \psi(s, z)>0\end{cases}
$$




$$
\begin{aligned}
& \alpha^{+}=(\max (0, \alpha))^{k} \\
& \alpha^{-}=(\max (0,-\alpha))^{k}
\end{aligned}
$$

Using these definitions, the problem reads:

Given a state $s \in S$, and functions

$\phi: S \times \mathbb{R}^{m+n} \rightarrow \mathbb{R}^{m}, \quad \psi: \mathbb{R}^{m} \rightarrow S \times \mathbb{R}^{n}$,

find policies and alphas $(z, \alpha) \in \mathbb{R}^{m} \times \mathbb{R}^{n}$,

s.t. $\phi\left(s, z, \alpha^{+}\right)=0, \psi(s, z)-\alpha^{-}=0$.

\section{Alternative Error Measure}

We now evaluate the same examples we have considered in the main text using a new error measure. The idea behind this measure is that one might be interested how far agent's interpolated consumption differs from the optimal solution at a certain point. Hence we do the following: Given a particular point in the state space, we compute the solution to the equilibrium problem using the interpolated equilibrium policy functions as tomorrow's policy. From that solution, we get consumption values for all three agents that we now compare to the interpolated consumption values. In spirit of the Euler Error we compute the relative deviation of the interpolated policy from the optimal solution. Hence, the error is given by $E=\left(\frac{c_{i n t}}{c_{\text {opt }}}-1\right)$.

Bond Economy with Three Agents

\begin{tabular}{||c||c|c||c|c||c|c||}
\hline \hline \multicolumn{1}{||c||}{} & \multicolumn{3}{c||}{} & \multicolumn{2}{c||}{ E state space } & \multicolumn{2}{c||}{ E equilibrium path } \\
\hline$\underline{b}$ & points & time $(\min )$ & $\max \mathrm{E}$ & $\varnothing \mathrm{E}$ & $\max \mathrm{E}$ & $\varnothing \mathrm{E}$ \\
\hline \hline 0.1 & $\mathbf{4 0}(45)$ & $\mathbf{0 . 5}(0.4)$ & $\mathbf{- 3 . 2}(-2.6)$ & $\mathbf{- 4 . 1}(-3.5)$ & $\mathbf{- 3 . 2}(-2.6)$ & $\mathbf{- 4 . 7}(-4.0)$ \\
\hline 0.1 & $\mathbf{1 1 3}(120)$ & $\mathbf{1 . 1}(1.0)$ & $\mathbf{- 3 . 3}(-2.5)$ & $\mathbf{- 4 . 5}(-3.6)$ & $\mathbf{- 3 . 3}(-2.5)$ & $\mathbf{- 5 . 1}(-4.2)$ \\
\hline 1.0 & $\mathbf{1 8 5}(190)$ & $\mathbf{6 . 5}(4.5)$ & $\mathbf{- 2 . 3}(-1.7)$ & $\mathbf{- 3 . 2}(-3.0)$ & $\mathbf{- 2 . 5}(-1.7)$ & $\mathbf{- 3 . 2}(-2.7)$ \\
\hline 1.0 & $\mathbf{9 4 1}(946)$ & $\mathbf{1 3}(11)$ & $\mathbf{- 3 . 3}(-2.3)$ & $\mathbf{- 4 . 5}(-3.6)$ & $\mathbf{- 3 . 3}(-2.5)$ & $\mathbf{- 5 . 1}(-3.3)$ \\
\hline \hline
\end{tabular}

Table D.1: Accuracy of adaptive grid (equidistant grid in brackets) 
Bond Economy with Four Agents

\begin{tabular}{||c||c|c||c|c||c|c||}
\hline \hline \multicolumn{1}{||c||}{} & \multicolumn{2}{c||}{} & \multicolumn{2}{c||}{ E state space } & \multicolumn{2}{c||}{ E equilibrium path } \\
\hline$\underline{b}$ & points & time $(\min )$ & $\max \mathrm{E}$ & $\varnothing \mathrm{E}$ & $\max \mathrm{E}$ & $\varnothing \mathrm{E}$ \\
\hline \hline 0.1 & $\mathbf{1 1 2}(120)$ & $\mathbf{4 . 5}(4)$ & $\mathbf{- 2 . 9}(-1.9)$ & $\mathbf{- 3 . 5}(-2.7)$ & $\mathbf{- 2 . 9}(-2.0)$ & $\mathbf{- 4 . 0}(-3.0)$ \\
\hline 1.0 & $\mathbf{9 1 4}(969)$ & $\mathbf{6 0}(51)$ & $\mathbf{- 1 . 9}(-1.5)$ & $\mathbf{- 2 . 7}(-2.6)$ & $\mathbf{- 1 . 9}(-1.5)$ & $\mathbf{- 2 . 8}(-3.4)$ \\
\hline \hline
\end{tabular}

Table D.2: Accuracy of adaptive grid (equidistant grid in brackets)

Bond and Stock Economy

\begin{tabular}{||c||c|c||c|c||c|c||}
\hline \hline \multicolumn{1}{||c||}{} & \multicolumn{2}{c||}{} & \multicolumn{2}{c||}{ E state space } & \multicolumn{2}{c||}{ E equilibrium path } \\
\hline & points & time $(\min )$ & $\max \mathrm{E}$ & $\varnothing \mathrm{E}$ & $\max \mathrm{E}$ & $\varnothing \mathrm{E}$ \\
\hline \hline & $\mathbf{1 2 3 5}(1250)$ & $\mathbf{3 1 0}(260)$ & $\mathbf{- 2 . 1}(-1.8)$ & $\mathbf{- 3 . 5}(-3.4)$ & $\mathbf{- 2 . 1}(-1.8)$ & $\mathbf{- 3 . 6}(-3.4)$ \\
\hline \hline
\end{tabular}

Table D.3: Accuracy of adaptive grid (equidistant grid in brackets) 